\title{
Left-sided Catamenial Pneumothorax: A Rare Clinical Entity
}

\author{
Naureen Narula ${ }^{1}$, Sam Ngu $^{1}$, Akshay Avula ${ }^{2}$, Wissam Mansour ${ }^{2}$, Michel Chalhoub ${ }^{3}$ \\ 1. Internal Medicine, Staten Island University Hospital, Staten Island, USA 2. Pulmonary and Critical Care Medicine, \\ Staten Island University Hospital, Northwell Health, Staten Island, USA 3. Pulmonary Medicine and Critical Care, \\ Staten Island University Hospital, Northwell Health, Staten Island, USA
}

Corresponding author: Naureen Narula,drnaureennarula@gmail.com

\begin{abstract}
Catamenial pneumothorax $(\mathrm{CP})$ is an extremely rare pulmonary pathology seen in women of reproductive age, typically occurring within 72 hours from the onset of menstrual bleeding. Multiple theories have been proposed to explain the etiopathogenesis of $\mathrm{CP}$; however, the exact underlying mechanism remains elusive. More than $90 \%$ of reported cases in the literature describe a right-sided presentation of pneumothorax. In this case report, we describe a rare left-sided presentation of CP and discuss the current literature on underlying etiopathogenesis, diagnostics, and available therapeutic modalities for managing this rare clinical entity.
\end{abstract}

Categories: Internal Medicine, Miscellaneous, Pulmonology

Keywords: catamenial pneumothorax

\section{Introduction}

The presence of endometrial tissue outside the uterine cavity is termed endometriosis while its presence inside the thoracic cavity is known as thoracic endometriosis [1-2]. Thoracic endometriosis syndrome includes four distinct clinical presentations: catamenial pneumothorax (CP), catamenial hemothorax, hemoptysis, and pulmonary nodules [3]. CP is a rare form of spontaneous pneumothorax (PTX) that occurs predominantly in women of reproductive age, typically within 72 hours from the onset of menstrual bleeding [1-3]. In 1958, Maurer et al. described the first case of recurrent PTX associated with concomitant menstruation [4]. Later in 1972, Lillington et al. [5] successfully coined the definition of CP. Current literature estimates suggest that CP accounts for up to one-third of surgically treated cases of PTX in women. It is the most common manifestation of thoracic endometriosis syndrome (TES). More than $90 \%$ of cases report a right-sided predilection for PTX. In this report, we describe an unusual presentation of leftsided CP and discuss the underlying etiopathogenesis and the available diagnostic and therapeutic modalities for managing $\mathrm{CP}$.

Received 04/23/2018 Review began 04/27/2018 Review ended 04/30/2018 Published 05/02/2018

๑) Copyright 2018 Narula et al. This is an open access article distributed under the terms of the Creative Commons Attribution License CC-BY 3.0., which permits unrestricted use, distribution, and reproduction in any medium, provided the original author and source are credited.

\section{Case Presentation}

A previously healthy, 24-year-old female presented to the emergency department (ED) with a one-day history of shortness of breath (SOB) and left-sided pleuritic chest pain. She complained experiencing progressively worsening dyspnea with physical activity. SOB was associated with left-sided chest pain described as stabbing in character, Th pain rated 6/10 in intensity on the pain scale and was non-radiating. No pertinent medical, surgical, or social history was noted. She denied a recent history of trauma. The patient was on the second day of her menstrual cycle. In the ED, her vitals showed a blood pressure of 125/70 $\mathrm{mmHg}$, a heart rate of 102 beats/minute and a respiratory rate of 19 breaths/min. She was afebrile and was maintaining an oxygen saturation of $99 \%$ on room air. On physical examination, she had diminished breath sounds on the left side. Chest X-ray (CXR) showed a moderate-sized left PTX with a $2.6 \mathrm{~cm}$ gap but without a mediastinal shift (Figure 1). 


\section{Cureus}

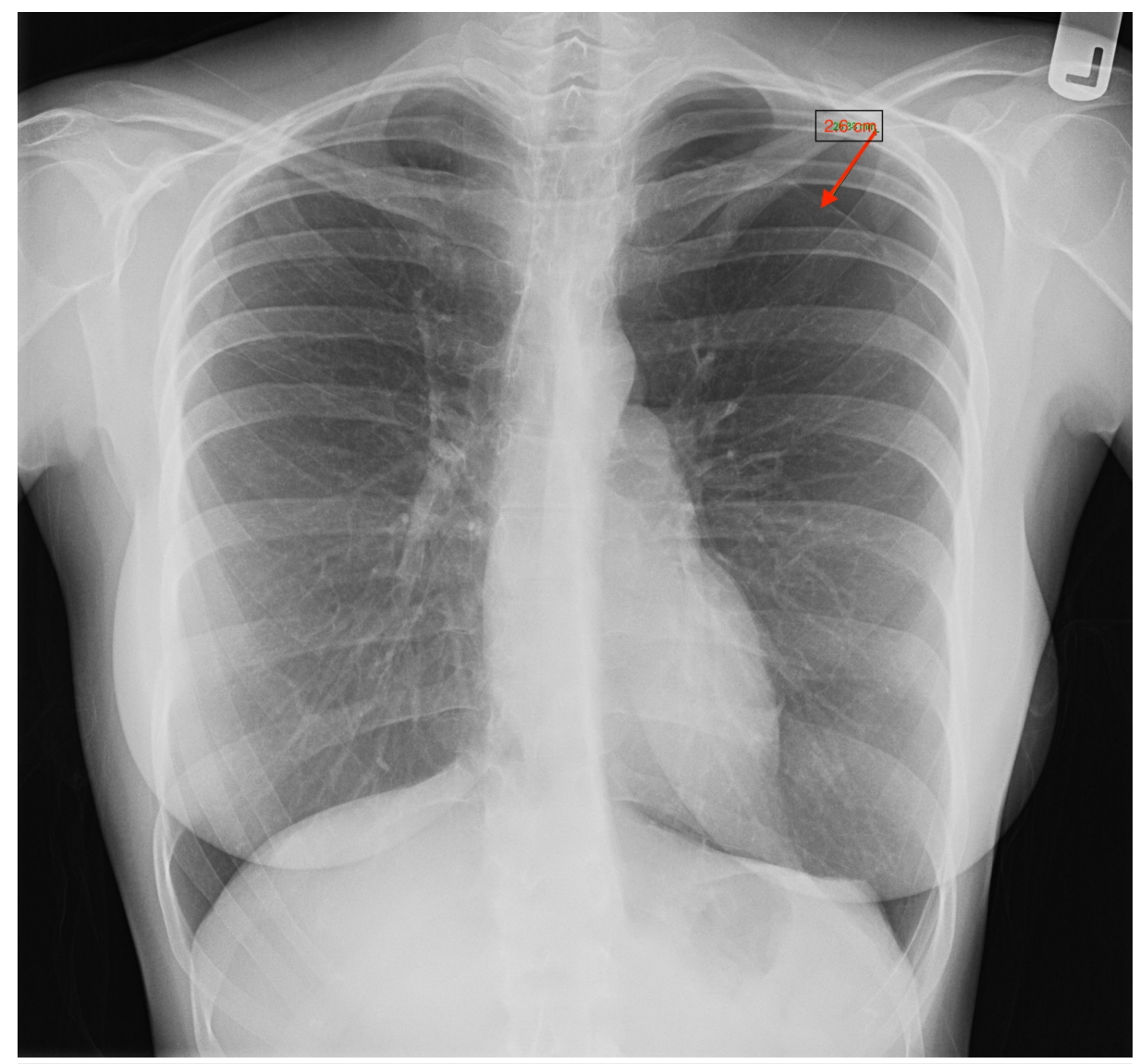

FIGURE 1: Chest x-ray

Chest x-ray (CXR) showing a moderate-sized left pneumothorax with a $2.6 \mathrm{~cm}$ gap.

Needle aspiration with the placement of a left-sided pigtail catheter was performed immediately. Blood work and basic metabolic chemistry were within the normal limits. Venous blood gas was grossly normal with the exception of lactic acidosis $(2.8 \mathrm{mmol} / \mathrm{L})$. For her pain, the patient received analgesics and symptomatic improvement was noted. A repeat CXR showed a decrease in the size of the PTX from 2.6 $\mathrm{cm}$ to $1.9 \mathrm{~cm}$. A consecutive CXR over the next two days showed significant improvement. A complete resolution of PTX was noted eventually. Consultants from pulmonary and obstetrics/gynecology confirmed the clinical diagnosis of catamenial-pneumothorax possibly associated with thoracic endometriosis. She was eventually discharged on day five of hospitalization on an oral contraceptive medication containing ethinyl estradiol and levonorgestrel.

\section{Discussion}

Thoracic endometriosis syndrome (TES) is characterized by the presence of endometrial tissue in or around the lung, and it consists of four distinct clinical presentations: catamenial pneumothorax (CP), catamenial hemothorax, hemoptysis, and pulmonary nodules [3]. CP consists of 30\%-50\% of all cases of thoracic endometriosis syndrome (TES) [1]. A diagnosis of CP requires a high degree of clinical suspicion relating pulmonary symptoms temporally to the phase of menstrual cycle. According to the widely accepted endometrial implant theory, endometrial tissues in the thorax rapidly proliferate and, subsequently, lead to a necrosis of the tissue, causing chest pain along with dyspnea, dry cough, pneumothorax, and, sometimes, hemoptysis [1-2]. Despite a temporal relationship with menstruation, the exact pathogenesis of catamenial pneumothorax remains elusive. Multiple theories have been postulated to describe the etiopathogenesis. The theory of coelomic metaplasia, prostaglandin theory, theory of retrograde menstruation, passage of air through the diaphragm, and lymphatic/vascular microembolization are some of the few widely accepted hypothesis $[1,3,6]$. Pathogenesis may be multifactorial, as described by Bricelj K et al. In this study, endometrial implants were seen on video-assisted thoracoscopic surgery (VATS) in 59.3\% of all patients diagnosed with $\mathrm{CP}$, whereas diaphragmatic defects ranging $1 \mathrm{~mm}$ to $10 \mathrm{~mm}$ were reported in $57.0 \%$ of patients [7]. The possible explanation for a right-sided predilection may be related to the clockwise movement of endometrial tissue within the peritoneal fluid from the pelvis along the right paracolic gutter to the subphrenic space. Two forces contribute to this movement: the falciform ligament preventing the movement of endometrial tissue to the left and the intraperitoneal pressure variations during respiration causing the right hemidiaphragm to contract against liver. This generates a piston-like effect, which 


\section{Cureus}

potentiates the endometrial tissue movement across the right hemidiaphragm [8].

CXR and a chest computed tomography (CT) scan have a limited role in delineating the endometrial source of origin of pneumothorax. CT chest may show associated pneumoperitoneum or diaphragmatic endometrial implants. Chest magnetic resonance imaging (MRI) may help in identifying hyperintense diaphragmatic and pleural endometriosis lesions on T1- and T2-weighted images [1]. However, direct visualization with VATS and histopathologic confirmation is considered the gold standard diagnostic modality [9]. Bagan et al. reported elevated serum levels of CA-125 as a possible diagnostic aid; however, CA-125 levels lack specificity; thus, routine monitoring of the levels is not recommended [10]. While there are no prospective trials comparing various management strategies, the treatment modalities for CP remain similar to that for endometriosis. Hormonal treatment is the cornerstone medical management therapy. It blocks feedback to the endometrial tissue and, therefore, prevents further dissemination. Oral contraceptives, danazol, and gonadotropin-releasing hormone (GnRH) analogs are commonly used therapeutics for managing CP [11]. Recently, dienogest - a synthetic oral progestogen, has shown potential benefits in preventing the recurrence of CP [12]. The surgical options include hysterectomy with bilateral salpingo-oophorectomy. Surgery is considered a definitive treatment and is usually reserved for severe refractory cases [6]. VATS-assisted resection of ectopic endometrial implants and the repair of diaphragmatic and pleural defects can theoretically lead to disease resolution. However, several cases of disease recurrence have been reported despite a repair of diaphragmatic defects [7]. A multidisciplinary approach collaborating pulmonary and gynecological consultation is considered ideal as the possibility of concomitant uterine endometriosis is fairly high in patients with CP.

Table 1 summarizes various theories explaining the etiopathogenesis of CP.

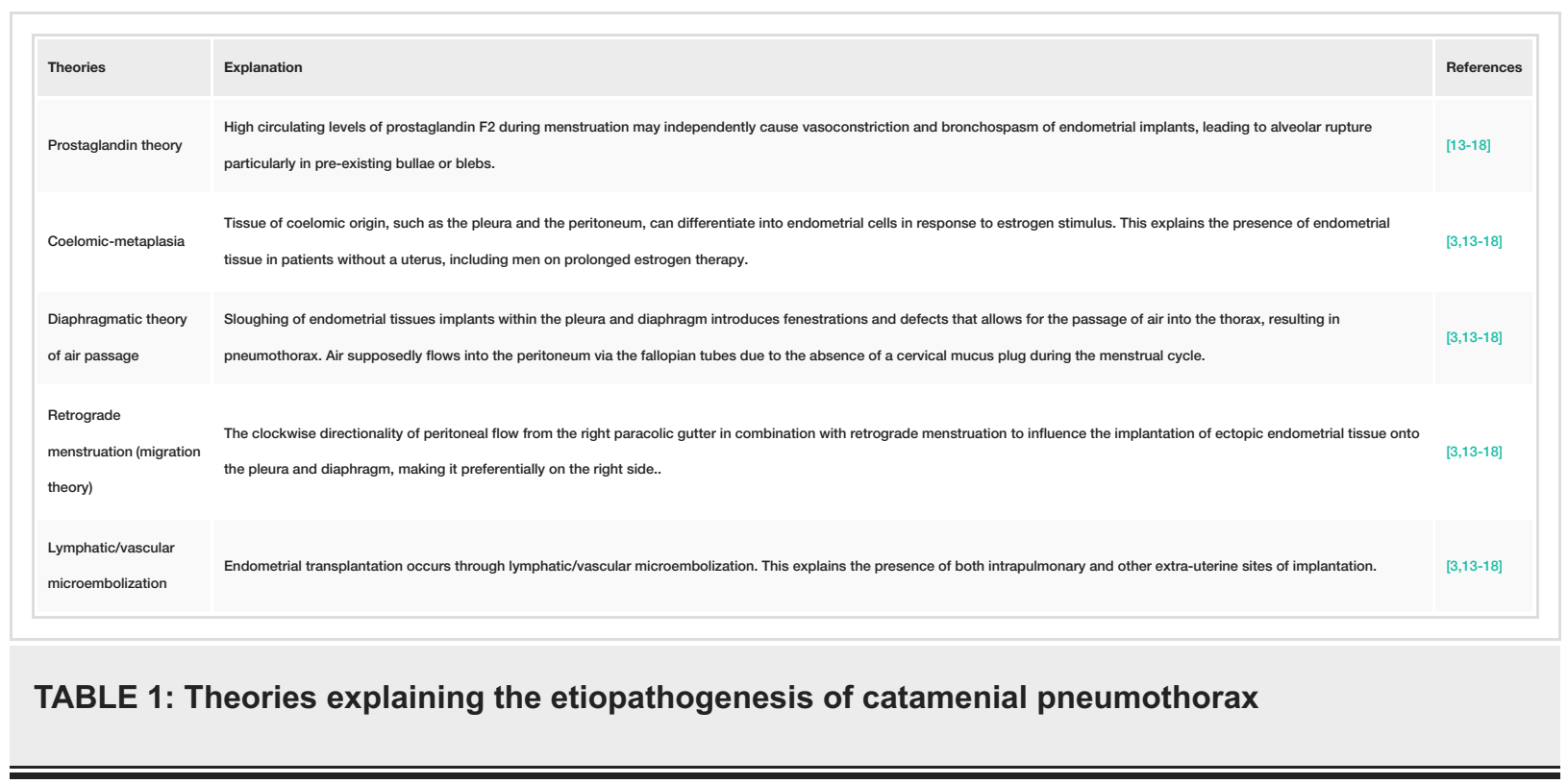

Conclusions

The diagnosis of CP requires a high degree of clinical suspicion. Diagnosis should be suspected in the presence of clinical signs of respiratory distress correlating temporaly with the menstrual cycle. Clinicians should not exclude diagnosis in left-sided presentations. The treatment may be either medical and/or surgical with the primary aim of avoiding disease recurrence. More longitudinal studies are required for formulating diagnostic and therapeutic guidelines for managing CP.

\section{Additional Information}

\section{Disclosures}

Human subjects: Consent was obtained by all participants in this study. SIUH Institutional Review Board issued approval Verbally obtained. Verbal consent was obtained. . Conflicts of interest: In compliance with the ICMJE uniform disclosure form, all authors declare the following: Payment/services info: All authors have declared that no financial support was received from any organization for the submitted work. Financial relationships: All authors have declared that they have no financial relationships at present or within the previous three years with any organizations that might have an interest in the submitted work. Other relationships: All authors have declared that there are no other relationships or activities that could appear to have influenced the submitted work.

\section{References}


1. Aissa S, Benzarti W, Alimi F, et al.: Catamenial pneumothorax revealing diaphragmatic endometriosis: a case report and revue of literature. Pan Afr Med J. 2017, 27:112. 10.11604/pamj.2017.27.112.8007

2. Inam, H, Inam S, Tahir M: Catamenial pneumothorax: a case report. J Pak Med Assoc. 2016, 66:1327-1329.

3. Alifano M, Trisolini R, Cancellieri A, Regnard JF: Thoracic endometriosis: current knowledge. Ann Thorac Surg. 2006, 81:761-769. 10.1016/j.athoracsur.2005.07.044

4. Maurer ER, Schaal JA, Mendez FL Jr: Chronic recurring spontaneous pneumothorax due to endometriosis of the diaphragm. J Am Med Assoc. 1958, 168:2013-2014. 10.1001/jama.1958.63000150008012c

5. Lillington GA, Mitchell SP, Wood GA: Catamenial pneumothorax. JAMA. 1972, 219:1328-1332. 10.1001/jama.1972.03190360038009

6. Arunthari V, Sevin B, Krishna M, Johnson MM: Catamenial pneumothorax with umbilical and diaphragmatic endometriosis: a case report and review of the literature. South Med J. 2008, 101:1043-1050. 10.1097/SMJ.0b013e31817bf9e1

7. Bricelj K, Srpcic M, Razem A, Snoj Z: Catamenial pneumothorax since introduction of video-assisted thoracoscopic surgery. Wien Klin Wochenschr. 2017, 129:717-726. 10.1007/s00508-017-1237-4

8. Papafragaki D, Concannon L: Catamenial pneumothorax: a case report and review of the literature. J Womens Health Larchmt. 2008, 17:367-372. 10.1089/jwh.2007.0553

9. Wong I, Afzal A, Gulkarov I, Chang R, Reyes A, Worku B: Recurrent pneumothoraces: making the link to catamenial pneumothorax. Am J Med. 2017, 130:6. 10.1016/j.amjmed.2016.12.047

10. Bagan P, Berna P, Assouad J, Hupertan V, Le Pimpec Barthes F, Riquet M: Value of cancer antigen 125 for diagnosis of pleural endometriosis in females with recurrent pneumothorax. Eur Respir J. 2008, 31:140-142. 10.1183/09031936.00094206

11. Beranger F, de Lesquen H, Goin G, Natale C, Bonnet PM, Avaro JP: Catamenial pneumothorax: easy to recognize, difficult to treat [Article in English, French]. Rev Pneumol Clin. 2014, 70:127-132. 10.1016/j.pneumo.2013.09.004

12. Lalani S, Black A, Hodge M, Tulandi T, Chen I: Dienogest therapy as a treatment for catamenial pneumothorax: case report and review of hormonal options. J Obstet Gynaecol Can. 2017, 39:764-768. 10.1016/j.jogc.2017.01.014

13. Joseph J, Sahn SA: Thoracic endometriosis syndrome: new observations from an analysis of 110 cases . Am J Med. 1996, 100:164-170. 10.1016/S0002-9343(97)89454-5

14. Alifano M, Roth T, Broët SC, Schussler O, Magdeleinat P, Regnard JF: Catamenial pneumothorax: a prospective study. Chest. 2003, 124:1004-1008. 10.1378/chest.124.3.1004

15. Bagan P, Le Pimpec Barthes F, Assouad J, Souilamas R, Riquet M: Catamenial pneumothorax: retrospective study of surgical treatment. Ann Thorac Surg. 2003, 75:378-381. 10.1016/S0003-4975(02)04320-5

16. Leong AC, Coonar AS, Lang-Lazdunski L: Catamenial pneumothorax: surgical repair of the diaphragm and hormone treatment. Ann R Coll Surg Engl. 2006, 88:547-549. 10.1308/003588406X130732

17. Korom S, Canyurt H, Missbach A, et al.: Catamenial pneumothorax revisited: clinical approach and systematic review of the literature. J Thorac Cardiovasc Surg. 2004, 128:502-508. 10.1016/j.jtcvs.2004.04.039

18. Laws HL, Fox LS, Younger JB: Bilateral catamenial pneumothorax. Arch Surg. 1977, 112:627-628. 10.1001/archsurg.1977.01370050087015 